\title{
Phase Equilibria in the System Vanadium Oxide-Niobium Oxide
}

\author{
J. L. Waring and R. S. Roth
}

(December 14, 1964)

\begin{abstract}
The phase diagram for the pseudo-binary system $\mathrm{V}_{2} \mathrm{O}_{5}-\mathrm{Nb}_{2} \mathrm{O}_{5}$ was constructed from fusion characteristics and $\mathrm{x}$-ray diffraction data. Three compounds were postulated for the system. The compound $\mathrm{VNb}_{9} \mathrm{O}_{25}$ and $\mathrm{V}_{2} \mathrm{Nb}_{23} \mathrm{O}_{62}$ were found to melt incongruently at about $1290{ }^{\circ} \mathrm{C}$ and $1322{ }^{\circ} \mathrm{C}$ respectively. The third compound occurring at about 93 to 94 mole percent $\mathrm{Nb}_{2} \mathrm{O}_{5}$ melted incongruently at approximately $1332{ }^{\circ} \mathrm{C}$. Apparently, $\mathrm{Nb}_{2} \mathrm{O}_{5}$ accepted up to 5 mole percent $\mathrm{V}_{2} \mathrm{O}_{5}$ in solid solution, however, $\mathrm{V}_{2} \mathrm{O}_{5}$ did not accept any appreciable $\mathrm{Nb}_{2} \mathrm{O}_{5}$ in solid solution.

In addition, the $\mathrm{Nb}_{2} \mathrm{O}_{5}$ rich portions of the following binary systems were reinvestigated: $\mathrm{ZnO}-\mathrm{Nb}_{2} \mathrm{O}_{5}, \mathrm{NiO}-\mathrm{Nb}_{2} \mathrm{O}_{5}, \mathrm{Al}_{2} \mathrm{O}_{3}-\mathrm{Nb}_{2} \mathrm{O}_{5}, \mathrm{TiO}_{2}-\mathrm{Nb}_{2} \mathrm{O}_{5}$, and $\mathrm{ZrO}_{2}-\mathrm{Nb}_{2} \mathrm{O}_{5}$. In each case an $\mathrm{Nb}_{2} \mathrm{O}_{5}$ solid solution had been reported previously in excess of 90 mole percent $\mathrm{Nb}_{2} \mathrm{O}_{5}$. However, subsequent data indicated that these $\mathrm{Nb}_{2} \mathrm{O}_{5}$ solid solutions contained discrete compounds which are related to those found in the pseudo-binary $\mathrm{V}_{2} \mathrm{O}_{5}-\mathrm{Nb}_{2} \mathrm{O}_{5}$.
\end{abstract}

\section{Introduction}

In a number of previously reported phase studies of the general type $M_{x} \mathrm{O}_{y}-\mathrm{Nb}_{2} \mathrm{O}_{5}$, the existence of $\mathrm{Nb}_{2} \mathrm{O}_{5}$-rich solid solutions were postulated. These include $\mathrm{TiO}_{2}-\mathrm{Nb}_{2} \mathrm{O}_{5}, \quad \mathrm{ZrO}_{2}-\mathrm{Nb}_{2} \mathrm{O}_{5} \quad[1],{ }^{1} \mathrm{Al}_{2} \mathrm{O}_{3}-$ $\mathrm{Nb}_{2} \mathrm{O}_{5}[3,4], \mathrm{ZnO}-\mathrm{Nb}_{2} \mathrm{O}_{5}[2]$ and $\mathrm{NiO}-\mathrm{Nb}_{2} \mathrm{O}_{5}$ $[3,4]$.

When selected compositions in these systems were examined they were found to give $x$-ray powder diffraction patterns with marked similarity one to the other as well as to the stable high temperature form of $\mathrm{Nb}_{2} \mathrm{O}_{5}$.

However, it seemed unlikely that a solid solution with oxygen deficiencies could account for the differences in the x-ray pattern. In an attempt to determine whether these phases were solid solutions or discrete compounds the system $\mathrm{V}_{2} \mathrm{O}_{5}-\mathrm{Nb}_{2} \mathrm{O}_{5} \mathrm{~W}$ : selected to be studied, as cations of similar ionic radii and the same valence might be most expected to form a solid solution.

Goldschmidt [3] surveyed and reported the existence of two intermediate phases in the system: $\beta^{\prime}(\mathrm{Nb}, \mathrm{V})_{2} \mathrm{O}_{5}$ and a phase with a rutile-type structure, $a=4.633 \AA$, $c=3.021 \AA$. However, no systematic attempt to study the phase equilibrium relationships in air has been previously reported.

X-ray diffraction data, together with the determination of the melting points of the compounds and the solidus and liquidus temperatures at various compositions across the system have supplied data from which an equilibrium diagram has been constructed.

${ }^{1}$ Figures in brackets indicate the literature references at the end of this paper.

\section{Sample Preparation and Test Methods}

High purity grade niobium and vanadium pentoxides were used. Spectrographic analysis indicated less than 0.1 percent $\mathrm{Si}, 0.01$ percent $\mathrm{Fe}, \mathrm{Sn}$, and $\mathrm{Ti}$ and 0.001 percent $\mathrm{Ca}$ and $\mathrm{Mg}$ in the former and less than about 0.01 percent $\mathrm{Si}, 0.001$ percent $\mathrm{Ca}$ and $\mathrm{Cr}$ and 0.0001 percent $\mathrm{Ag}, \mathrm{Ca}, \mathrm{Mg}$ and $\mathrm{Mn}$ in the latter. $\mathrm{Cu}$ was not determined in $\mathrm{Nb}_{2} \mathrm{O}_{5}$ because of $\mathrm{Nb}$ interference.

Mixtures of $\mathrm{V}_{2} \mathrm{O}_{5}$ and $\mathrm{Nb}_{2} \mathrm{O}_{5}$ were weighed to the nearest $\pm 0.1 \mathrm{mg}$ in sufficient quantities to yield 2 $\mathrm{g}$ batches. No corrections were made for percent purity except loss on ignition. Each batch was mixed in a mechanical shaker for $15 \mathrm{~min}$. The mixtures were then pressed into a disk in a $5 / 8$ in. diameter mold at $10^{4} \mathrm{lb} \mathrm{in}^{-2}$. The disks were placed on setters fabricated from platinum foil and calcined in air at $500{ }^{\circ} \mathrm{C}$ for $10 \mathrm{hr}$. Following this preliminary heat treatment specimens ranging in composition from 5 to 55 mole percent $\mathrm{Nb}_{2} \mathrm{O}_{5}$ were recalcined at $600{ }^{\circ} \mathrm{C}$ for $168 \mathrm{hr}$ in sealed Pt tubes. Specimens ranging in composition from 90 to 99 mole percent $\mathrm{Nb}_{2} \mathrm{O}_{5}$ received an additional calcination of $1000{ }^{\circ} \mathrm{C}$ for $5 \mathrm{hr}$.

Since $\mathrm{V}_{2} \mathrm{O}_{5}$ was found to be volatile only to the extent of about 0.46 percent when heated to about $1000{ }^{\circ} \mathrm{C}$ for $6 \mathrm{hr}$, no correction other than loss on ignition was applied. However, to minimize possible volatility at higher temperatures, sealed platinum tubes were employed for all succeeding experiments unless otherwise stated. At this point it should be noted that platinum tubes approximately $2 \mathrm{~cm} \mathrm{long}$ were used to contain the specimen. About one-third 
of the volume was occupied by the specimen and the remainder of the tube was flattened prior to sealing. At elevated temperatures the expansion of the flattened portion of the tube provided the necessary additional volume for expansion of the vapors without rupture.

Sub-solidus and melting point values were obtained by quenching specimens sealed in platinum tubes and examining them at room temperature. An electrically heated vertical tube resistance furnace was used for quenching which had a primary winding of $60 \% \mathrm{Pt}-40 \% \mathrm{Rh}$ and a secondary or booster winding of $80 \% \mathrm{Pt}-20 \%$ Rh. An a-c controller utilizing the furnace primary as one arm of a Wheatstone bridge served to control the furnace temperature to $\pm 2{ }^{\circ} \mathrm{C}$ for extended periods of time. The secondary was regulated with a variable autotransformer.

Temperatures were measured with a $\mathrm{Pt}$ versus Pt 10 percent $R h$ thermocouple which was calibrated

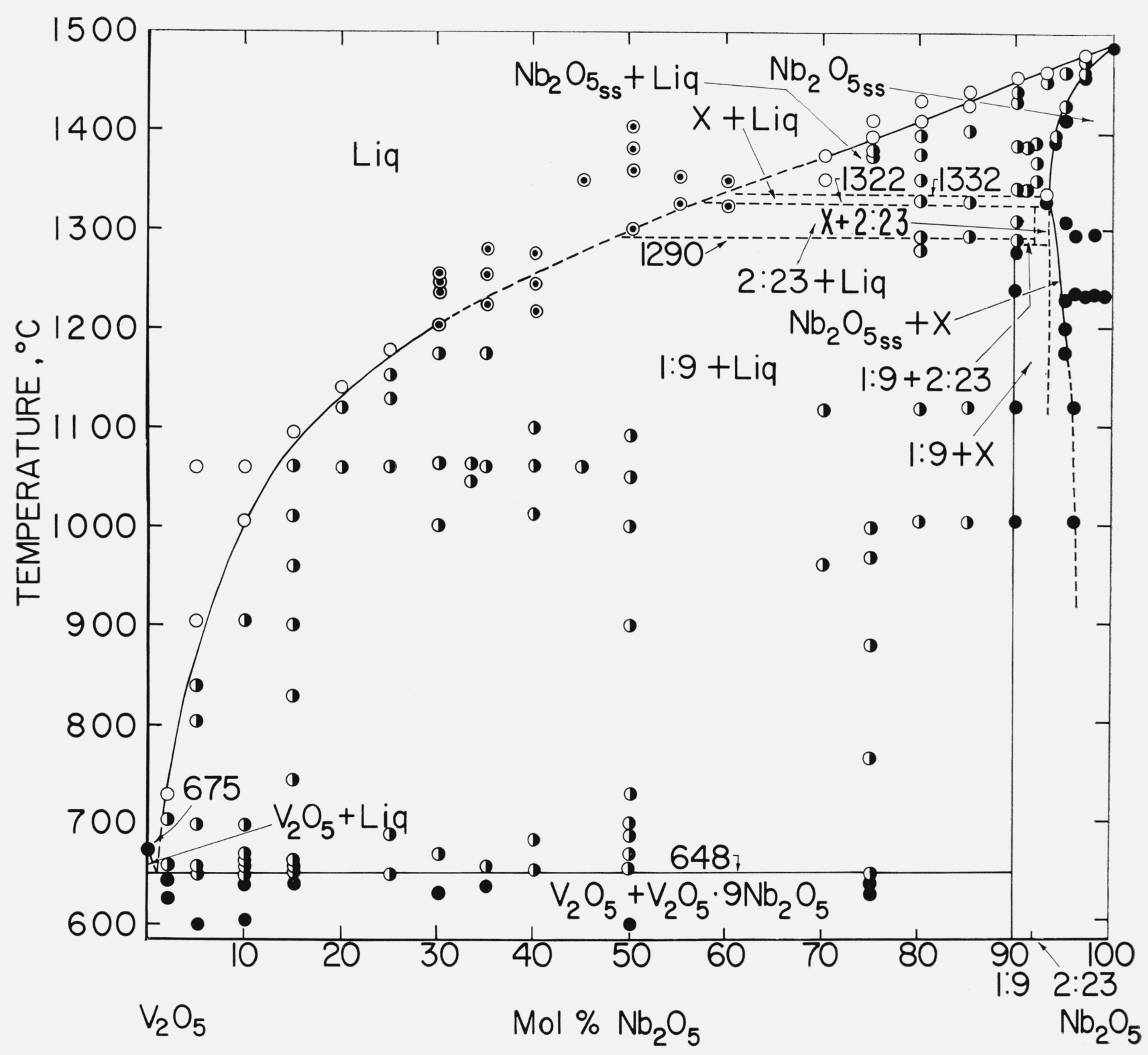

Figure 1. Phase equilibrium diagram for the system vanadium oxide-niobium-oxide.

-no melting.

- - partial melting.

-complete melting.

O-cannot be interpreted from fusion characteristics.

Liq.-liquid.
$\mathrm{X}$-unknown phase

For clarity, not all experimental data appearing in table 4 are plotted on this diagram. 
several times during the course of the work against the reported values of $\mathrm{NaCl}\left(800.5^{\circ} \mathrm{C}\right)$ [6], $\mathrm{Au}(1063$ $\left.{ }^{\circ} \mathrm{C}\right)$ [5] and barium disilicate $\left(1420{ }^{\circ} \mathrm{C}\right)$ [7].

Liquidus values were established in the following manner: (a) In the region from 2 to $5 \% \mathrm{Nb}_{2} \mathrm{O}_{5}$, the formation of a concave meniscus established the liquidus. (b) In the region from 5 to $30 \% \mathrm{Nb}_{2} \mathrm{O}_{5}$, specimens when quenched from above the liquidus and examined at room temperature were glass. (c) In the region from 30 to $70 \% \quad \mathrm{Nb}_{2} \mathrm{O}_{5}$, no criteria could be employed to establish the liquidus since any given composition between these limits gave the same physical appearance over a range of about a hundred degrees, and this portion (fig. 1) of the curve was dashed. (d) In the region from 70 to $100 \% \mathrm{Nb}_{2} \mathrm{O}_{5}$ (fig. 1) the formation of a concave meniscus and the abrupt change in $x$-ray diffraction pattern established the liquidus.

The first sign of glazing of the specimen's surface, together with a marked color change established the solidus in the $0-90$ mole percent $\mathrm{Nb}_{2} \mathrm{O}_{5}$ compositional range. This interpretation seemed reasonably valid since most specimens showed abrupt changes in x-ray diffraction patterns when partially melted. Unfortunately, compositions in the $\mathrm{Nb}_{2} \mathrm{O}_{5}$ rich portion of the system $90-99 \% \mathrm{Nb}_{2} \mathrm{O}_{5}$, did not provide a definitive picture of partial melting, and the approximate solidus values were delineated by data obtained from changes in the x-ray diffraction powder patterns of the quenched specimens and the solidus (figs. 1 and 2) in this region is dashed. The precision of the temperature measurement for the experimental data points is about $\pm 2{ }^{\circ} \mathrm{C}$ and the overall accuracy of the reported temperature is about $\pm 5{ }^{\circ} \mathrm{C}$.

Equilibrium is generally considered to have been obtained when x-ray diffraction patterns of specimens successively heated for longer times and/or at higher

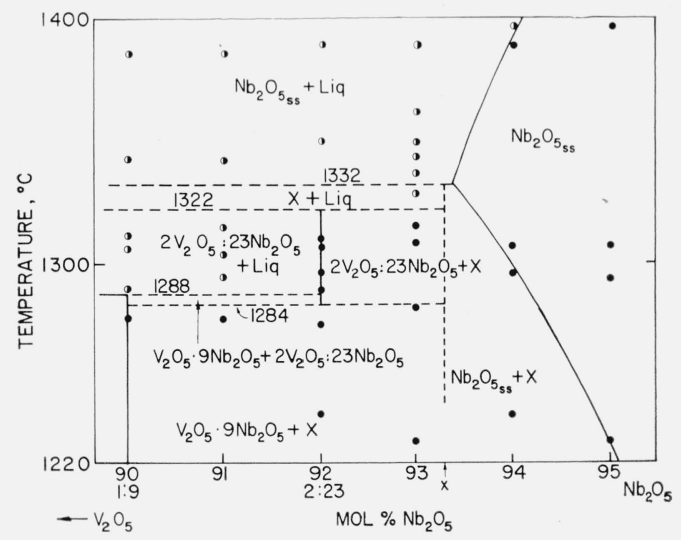

Figure 2. Expanded view of the high $\mathrm{Nb}_{2} \mathrm{O}_{5}$ region of the phase diagram for the system vanadium oxide-niobium oxide.

-no melting.

- partial melting.

Liq.--liquid.

$\mathrm{X}$-unknown phase

$\mathrm{Nb}_{2} \mathrm{O}_{588}-\mathrm{Nb}_{2} \mathrm{O}_{5}$ solid solution. temperatures show no change. However, as this system does not appear to be binary at high temperatures this criteria could not always be applied without interpretation. (See sec. 4 and table 4.) X-ray diffraction powder patterns were made using a high angle recording Geiger counter diffractometer and nickel-filtered copper radiation, with the Geiger counter traversing the specimen at $1 / 4^{\circ} 2 \theta / \mathrm{min}$ and radiation being recorded on the chart at $1^{\circ} 2 \theta / \mathrm{in}$. The unit cell dimensions reported can be considered accurate to about \pm 5 in the last decimal place listed.

\section{Compounds in the Pseudo-Binary System $\mathrm{V}_{2} \mathrm{O}_{5}-\mathrm{Nb}_{2} \mathrm{O}_{5}$}

\section{1. $\mathrm{V}_{2} \mathrm{O}_{5}$}

Holtzberg et al. [8] concluded from x-ray diffraction and differential thermal analyses data that $\mathrm{V}_{2} \mathrm{O}_{5}$ has only one stable form. These results were later independently verified by Levin and McDaniel [9] by high temperature x-ray diffraction analysis. The structure of $\mathrm{V}_{2} \mathrm{O}_{5}$ was described by Byström et al. [10] as having the space group Pmmn with two molecules per unit cell, and the indexed x-ray powder diffraction pattern was reported by Swanson et al. [11] $a=11.51 \AA, \quad b=3.559 \AA, c=4.371 \AA$. The melting point of $675 \pm 5{ }^{\circ} \mathrm{C}$ was established for $\mathrm{V}_{2} \mathrm{O}_{5}$ by C. L. McDaniel of this laboratory [12].

\subsection{Compound $\mathrm{V}_{2} \mathrm{O}_{5} \cdot 9 \mathrm{Nb}_{2} \mathrm{O}_{5}$}

The compound $\mathrm{V}_{2} \mathrm{O}_{5} \cdot 9 \mathrm{Nb}_{2} \mathrm{O}_{5}$ was found to occur in the present study and to be stable to the incongruent melting point at about $1290{ }^{\circ} \mathrm{C}$. Goldschmidt [3] reported a solid solution phase $\beta^{\prime}(\mathrm{Nb}, \mathrm{V})_{2} \mathrm{O}_{5}$ which had a nominal composition $\mathrm{Nb}_{1.8} \mathrm{~V}_{0.2} \mathrm{O}_{5}$. He reported the unindexed x-ray diffraction pattern of $\beta^{\prime}$ $(\mathrm{Nb}, \mathrm{V})_{2} \mathrm{O}_{5}$. In the present study, the x-ray diffraction powder pattern of $\mathrm{V}_{2} \mathrm{O}_{5} \cdot 9 \mathrm{Nb}_{2} \mathrm{O}_{5}$ was found to be essentially the same as that previously reported. The compound $\mathrm{V}_{2} \mathrm{O}_{5} \cdot 9 \mathrm{Nb}_{2} \mathrm{O}_{5}$ (table 1) which was indexed on the basis of a body-centered tetragonal cell $a=15.72 \AA, c=3.821 \AA$ was found to be isostructural with a series of phases of the general type $\mathrm{M}_{2} \mathrm{O}_{5} \cdot 9 \mathrm{M}_{2}{ }_{2} \mathrm{O}_{5}$ [13] and apparently with $\mathrm{Ta}_{2} \mathrm{O}_{5} \cdot 2 \mathrm{Nb}_{2} \mathrm{O}_{5}$ which was previously reported by Holtzberg and Reisman [14] as being cubic, and by Mohanty et al. [15] as being tetragonal.

\subsection{Compound $\mathrm{V}_{2} \mathrm{Nb}_{23} \mathrm{O}_{62}$}

A compound was found to occur in the system at the ratio $2 \mathrm{~V}_{2} \mathrm{O}_{5}: 23 \mathrm{Nb}_{2} \mathrm{O}_{5}$ which exists from about $1284{ }^{\circ} \mathrm{C}$, depending on the length of heating time, to the incongruent melting point at $1322{ }^{\circ} \mathrm{C}$. The indexed x-ray diffraction powder pattern given in table 2 has a marked similarity to that of the high temperature form of $\mathrm{Nb}_{2} \mathrm{O}_{5}$. However, this phase could not be indexed as a solid solution of the high temperature modification $\mathrm{Nb}_{2} \mathrm{O}_{5}$. Roth and Wadsley [16] have shown from a single crystal structure determination that a phase $\mathrm{TiO}_{2} \cdot 12 \mathrm{Nb}_{2} \mathrm{O}_{5}$ occurs in the system $\mathrm{TiO}_{2}-\mathrm{Nb}_{2} \mathrm{O}_{5}$ and is a member of a series 
$\mathrm{M}_{3 n+1} \mathrm{O}_{8 n-2}$ with $n=8$. The composition $2 \mathrm{~V}_{2} \mathrm{O}_{5}$. $23 \mathrm{Nb}_{2} \mathrm{O}_{5}$ was found to have an $\mathrm{x}$-ray diffraction powder pattern which is apparently isostructural with $\mathrm{TiO}_{2} \cdot 12 \mathrm{Nb}_{2} \mathrm{O}_{5}$ which could be indexed on the basis of a monoclinic cell $a=29.77 \AA, b=3.823 \AA, c=21.05$ A and $\beta=95^{\circ} 1.6^{\prime}$.

The composition $2 \mathrm{~V}_{2} \mathrm{O}_{5} \cdot 23 \mathrm{Nb}_{2} \mathrm{O}_{5}$ has the general formula $\mathrm{M}_{50} \mathrm{O}_{125}$. However, the structural formula $\mathrm{M}_{3 n+1} \mathrm{O}_{8 n-2}$ (referring to one-half the unit cell) requires the composition to be $\mathrm{M}_{50} \mathrm{O}_{124}$. From the above considerations it is likely that 1 out of 125 oxygen ions are lost and that the system is not binary.

TABLE 1. X-ray diffraction powder data for the compound $\mathrm{V}_{2} \mathrm{O}_{5} \cdot 9 \mathrm{Nb}_{2} \mathrm{O}_{5}(\mathrm{CuK} \alpha$ radiation $)$

\begin{tabular}{|c|c|c|c|c|}
\hline$d \operatorname{Obs} \AA$ & $I / I_{0}^{\mathrm{a}}$ & $1 / d^{2}$ obs $\AA^{-2}$ & $1 / d^{2}$ ealc. $\AA^{-2} \mathrm{~b}$ & $h k l \mathrm{~b}^{\mathrm{b}}$ \\
\hline $\begin{array}{c}11.340 \\
7.82 \\
5.54 \\
4.96 \\
3.92\end{array}$ & $\begin{array}{r}1 \\
1 \\
1 \\
25 \\
3\end{array}$ & $\begin{array}{r}0.0080 \\
.0163 \\
.0326 \\
.0407 \\
.0652\end{array}$ & $\begin{array}{r}0.0081 \\
.0162 \\
.0325 \\
.0406 \\
.0649\end{array}$ & $\begin{array}{l}110 \\
200 \\
220 \\
310 \\
400\end{array}$ \\
\hline $\begin{array}{l}\text { 3. } 70 \\
3.490 \\
3.353 \\
3.076 \\
2.868\end{array}$ & $\begin{array}{r}35 \\
100 \\
12 \\
1 \\
30\end{array}$ & $\begin{array}{l}.0729 \\
.0815 \\
.0889 \\
.1057 \\
.1216\end{array}$ & $\begin{array}{l}.0726 \\
.0811 \\
.0888 \\
.1055 \\
.1213\end{array}$ & $\begin{array}{l}101 \\
420 \\
211 \\
510 \\
312\end{array}$ \\
\hline $\begin{array}{l}2.696 \\
2.482 \\
2.426 \\
2.318 \\
2.222\end{array}$ & $\begin{array}{r}15 \\
15 \\
5 \\
5 \\
1\end{array}$ & $\begin{array}{l}.1376 \\
.1623 \\
.1700 \\
.1862 \\
.2025\end{array}$ & $\begin{array}{l}1375 \\
.1622 \\
.1699 \\
.1862 \\
.2028\end{array}$ & $\begin{array}{l}411 \\
620 \\
501 \\
521 \\
710\end{array}$ \\
\hline $\begin{array}{l}2.177 \\
2.139 \\
2.062 \\
1.996 \\
1.962\end{array}$ & $\begin{array}{r}1 \\
5 \\
21 \\
1 \\
2\end{array}$ & $\begin{array}{l}.2110 \\
.2186 \\
.2353 \\
.2510 \\
.2597\end{array}$ & $\begin{array}{l}.2109 \\
.2186 \\
.2353 \\
.2510 \\
.2596\end{array}$ & $\begin{array}{l}640 \\
611 \\
730 \\
631 \\
800\end{array}$ \\
\hline 1. 910 & 12 & .2742 & .2742 & 002 \\
\hline
\end{tabular}

a Relative intensity.

b Based on tetragonal cell, $a=15.72 \AA, c=3.821 \AA$.

TABLE 2. X-ray diffraction powder data for the compound $\mathrm{V}_{2} \mathrm{Nb}_{23} \mathrm{O}_{62}(\mathrm{CuK} \alpha$ radiat on $)$

\begin{tabular}{|c|c|c|c|c|}
\hline$d \mathrm{Obs}$ & $I / I_{0} \mathrm{a}$ & $1^{\mathrm{b}} / d^{2}$ obs $\AA^{-2}$ & $1^{\mathrm{b} / d^{2}}$ calc $\AA^{-2}$ & $h k l$ \\
\hline$\AA$ & & & & \\
\hline 10. 48 & 4 & 0.0091 & 0.0092 & 002 \\
\hline 9. 04 & 3 & .0125 & .0125 & $\overline{202}$ \\
\hline 6.81 & 3 & .0216 & .0216 & 401 \\
\hline 5.25 & 3 & .0364 & .0364 & 004 \\
\hline 5. 09 & 30 & .0387 & .0387 & $\overline{2} 04$ \\
\hline 4. 70 & 20 & .0450 & .0449 & 601 \\
\hline 3.74 & 43 & .0716 & .0716 & 111 \\
\hline 3.60 & 100 & .0772 & .0773 & 801 \\
\hline 3. 469 & 80 & .0831 & $\begin{array}{r}0831 \\
0.0892\end{array}$ & $\frac{206}{113}$ \\
\hline 3. 348 & 17 & .0892 & $\{.0893$ & $\frac{1}{605}$ \\
\hline 3. 273 & 5 & .0933 & $\begin{array}{r}.0895 \\
.0933\end{array}$ & $\begin{array}{l}\frac{312}{406} \\
\end{array}$ \\
\hline 3. 213 & 5 & .0969 & $\{.0966$ & 313 \\
\hline 3.068 & 5 & .1061 & $\begin{array}{l}.0909 \\
.1062\end{array}$ & $\begin{array}{l}510 \\
605\end{array}$ \\
\hline 2. 839 & 23 & 1241 & .1242 & 710 \\
\hline 2. 761 & 17 & .1312 & .1313 & 315 \\
\hline 2. 700 & 20 & .1372 & 1372 & 712 \\
\hline 2. 620 & 3 & .1459 & $\left\{\begin{array}{l}.1456 \\
1457\end{array}\right.$ & $\frac{008}{208}$ \\
\hline 2.528 & 27 & .1565 & $\begin{array}{l}.1406 \\
.1564\end{array}$ & 1005 \\
\hline 2. 467 & 16 & .1643 & $\left\{\begin{array}{l}1642 \\
1645\end{array}\right.$ & $\frac{607}{9} 12$ \\
\hline 2. 310 & 17 & .1874 & $\begin{array}{r}.1645 \\
.1872\end{array}$ & $\begin{array}{l}912 \\
516\end{array}$ \\
\hline 2. 066 & 37 & .2343 & .2344 & $\begin{array}{llll}\overline{4} & 0 & 10\end{array}$ \\
\hline 2. 039 & 38 & .2404 & .2403 & 609 \\
\hline 1. 913 & 33 & .2734 & .2736 & 020 \\
\hline
\end{tabular}

a Relative intensity.

b Based on monoclinic cell $a=29.77 \AA, b=3.823 \AA, c=21.05 \AA$ and $\beta=95^{\circ} 1.6^{\prime}$.

\subsection{Compound X}

A new phase was found to occur in the system at approximately 93 to 94 mole percent $\mathrm{Nb}_{2} \mathrm{O}_{5}$ and to melt incongruently at about $1332{ }^{\circ} \mathrm{C}$. The partially indexed x-ray diffraction powder pattern is given in table 3 . The $x$-ray pattern of this phase has the greatest similarity to the high temperature polymorph of $\mathrm{Nb}_{2} \mathrm{O}_{5}$ of any of the phases which occur in the system. However, the low angle $x$-ray diffraction lines, which should shift the least in $2 \theta$ for a solid solution, actually are shifted more than many high angle lines. Therefore, the pattern cannot be indexed on the basis of an $\mathrm{Nb}_{2} \mathrm{O}_{5}$ solid solution or as a solid solution of $\mathrm{V}_{2} \mathrm{Nb}_{23} \mathrm{O}_{62}$.

TABLE 3. X-ray diffraction powder data for the unknown phase $X$ occurring at about 93 to 94 mole percent $\mathrm{Nb}_{2} \mathrm{O}_{5}$ ( $\mathrm{CuK} \alpha$ radiation)

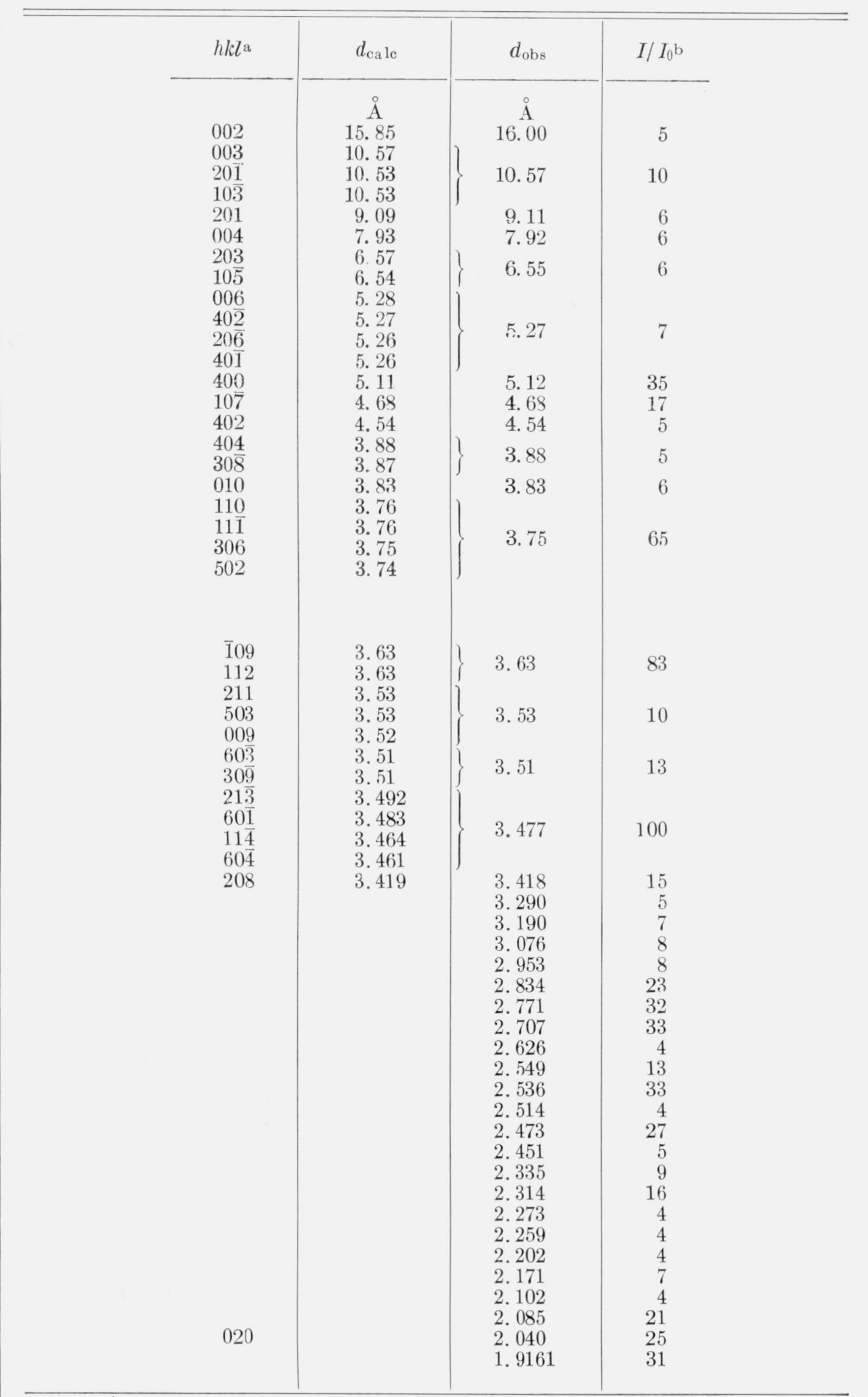

a This pattern was tentatively indexed only after approximate $a, c$, and $\beta$ unit cell constants were provided by Mr. A. Perloff from $h 0 l$ single crystal precession data. The unit cell constants derived from this data are $a=21.12 \mathrm{~A}, b=3.831 \mathrm{~A}$, $c=32.75 \AA$ and $\boldsymbol{\beta}=104^{\circ} 5^{\prime}$. With the exception of the (020) Miller indexes are not reported for reflections with $d$ values less than $3.419 \AA$ because of the relatively large size of the monoclinic cell. 


\subsection{Rutile Type Phase}

Goldschmidt [3] reported a phase with a rutile type structure and an approximate composition ranging from $90 \mathrm{~V}_{2} \mathrm{O}_{5}: 10 \mathrm{Nb}_{2} \mathrm{O}_{5}$ to about $75 \mathrm{~V}_{2} \mathrm{O}_{5}$ : $25 \mathrm{Nb}_{2} \mathrm{O}_{5}$. According to Goldschmidt this rutile phase formed from specimens fused in a carbon arc furnace in an atmosphere of argon and was not present when the specimens were annealed at $800{ }^{\circ} \mathrm{C}$ in air. In the present study no rutile phase was found to occur in the system in an air environment. However, the composition $75 \mathrm{~V}_{2} \mathrm{O}_{5}: 25 \mathrm{Nb}_{2} \mathrm{O}_{5}$ heated to about $1300{ }^{\circ} \mathrm{C}$ in a flowing stream of argon contained a rutile type phase.

From the above discussion it can probably be concluded the rutile phase is a reduced phase which occurs in the $\mathrm{V}-\mathrm{Nb}-\mathrm{O}$ system.

\section{6. $\mathrm{Nb}_{2} \mathrm{O}_{5}$}

The phase equilibrium relations of the polymorphs of $\mathrm{Nb}_{2} \mathrm{O}_{5}$ have been discussed by various workers [17], [18], [19], [20]. Since it has been concluded by $[17],[20],[21]$ that the high temperature polymorph of $\mathrm{Nb}_{2} \mathrm{O}_{5}$ is the stable form and since $\mathrm{V}_{2} \mathrm{O}_{5}$ has no catalytic action on the various modifications of $\mathrm{Nb}_{2} \mathrm{O}_{5}$ no phase transition has been indicated. The unit cell dimensions were reported previously by one of the present authors [21].

\section{Discussion of Phase Equilibria}

Although the $\mathrm{V}_{2} \mathrm{O}_{5}-\mathrm{Nb}_{2} \mathrm{O}_{5}$ system does not appear to be binary, a pseudo-binary phase diagram can be constructed from the experimental data listed in table 4. The appearance of the pseudo-binary diagram might well be changed by different environmental and/or time-temperature experimental conditions and only a complete study of the ternary diagram $\mathrm{V}-\mathrm{Nb}-\mathrm{O}$ could be expected to elucidate the true equilibrium relations. As constructed, the pseudo-binary system contains three intermediate compounds, all melting incongruently. The indexed x-ray diffraction powder pattern and unit cell dimensions of the first, $\mathrm{V}_{2} \mathrm{O}_{5} \cdot 9 \mathrm{Nb}_{2} \mathrm{O}_{5}$, are given in table 1 and those of the second, $\mathrm{V}_{2} \mathrm{Nb}_{23} \mathrm{O}_{62}$, in table 2 . The $d$-spacings for the third, a compound of unknown symmetry occurring at about 93 to 94 mole percent $\mathrm{Nb}_{2} \mathrm{O}_{5}$, are given in table 3 .

TABLE 4. Experimental data for compositions in the system vanadium oxide-niobum oxide

\begin{tabular}{|c|c|c|c|c|c|}
\hline \multicolumn{2}{|c|}{ Composition } & \multicolumn{2}{|c|}{ Heat treatment a } & \multicolumn{2}{|r|}{ Results } \\
\hline $\mathrm{V}_{2} \mathrm{O}_{5}$ & $\mathrm{Nb}_{2} \mathrm{O}_{5}$ & Temp. & Time & Physical observation & $\mathrm{X}$-ray diffraction analysis $b$ \\
\hline $\begin{array}{c}\text { Mole \% } \\
98\end{array}$ & $\begin{array}{c}\text { Mole } \% \\
2\end{array}$ & $\begin{array}{r}{ }^{\circ} C \\
627 \\
649 \\
661 \\
707 \\
730\end{array}$ & $\begin{array}{l}h r \\
1 \\
\quad 0.08 \\
.08 \\
.25 \\
.33\end{array}$ & $\begin{array}{l}\text { Not melted } \\
\text { Partially melted. } \\
\text { Completely melted }\end{array}$ & $\begin{array}{l}\mathrm{V}_{2} \mathrm{O}_{5}+\mathrm{V}_{2} \mathrm{O}_{5} \cdot 9 \mathrm{Nb}_{2} \mathrm{O}_{5}+\text { unknown }{ }^{\mathrm{c}} \\
\mathrm{V}_{2} \mathrm{O}_{588} \mathrm{~d}+\mathrm{V}_{2} \mathrm{O}_{5} \cdot 9 \mathrm{Nb}_{2} \mathrm{O}_{5} \\
\mathrm{~V}_{2} \mathrm{O}_{5_{88}}{ }^{\mathrm{d}}+\mathrm{unknown}^{\mathrm{c}} \\
\quad \text { Do. }\end{array}$ \\
\hline 95 & 5 & $\begin{array}{r}600 \\
602 \\
652 \\
658 \\
700 \\
804 \\
841 \\
904 \\
1060\end{array}$ & $\begin{array}{r}.3 \\
19 \\
.5 \\
.5 \\
.5 \\
.5 \\
.5 \\
1\end{array}$ & $\begin{array}{l}\begin{array}{l}\text { Not melted } \\
\text { do }\end{array} \\
\text { Partially melted } \\
\text { do } \\
\text { Completely melted do }\end{array}$ & $\begin{array}{l}\mathrm{V}_{2} \mathrm{O}_{5}+\mathrm{L}-\mathrm{Nb}_{2} \mathrm{O}_{5} \mathrm{e} \\
\text { Do. } \\
\mathrm{V}_{2} \mathrm{O}_{5} \cdot 9 \mathrm{Nb}_{2} \mathrm{O}_{5}+\mathrm{V}_{2} \mathrm{O}_{588} \mathrm{~d} \\
\text { Do. } \\
\text { Do. } \\
\mathrm{V}_{2} \mathrm{O}_{588} \mathrm{~d} \\
\text { Do. } \\
\text { Glass } \\
\text { Do. }\end{array}$ \\
\hline 90 & 10 & $\begin{array}{r}605 \\
640 \\
650 \\
652 \\
658 \\
662 \\
664 \\
666 \\
673 \\
700 \\
904 \\
1006 \\
1060\end{array}$ & $\begin{array}{r}.5 \\
20 \\
.33 \\
.33 \\
.33 \\
67 \\
.33 \\
.33 \\
.08 \\
.5 \\
.5 \\
.5 \\
1\end{array}$ & $\begin{array}{l}\text { Not melted } \\
\text { Partially melted } \\
\text { Pald }\end{array}$ & $\begin{array}{l}\mathrm{V}_{2} \mathrm{O}_{5}+\mathrm{V}_{2} \mathrm{O}_{5} \cdot 9 \mathrm{Nb}_{2} \mathrm{O}_{5} \\
\mathrm{~V}_{2} \mathrm{O}_{5_{s 8}}{ }^{\mathrm{d}}+\mathrm{V}_{2} \mathrm{O}_{5} \cdot 9 \mathrm{Nb}_{2} \mathrm{O}_{5} \\
\mathrm{~V}_{2} \mathrm{O}_{5_{48}}{ }^{\mathrm{d}}+\mathrm{V}_{2} \mathrm{O}_{5} \cdot 9 \mathrm{Nb}_{2} \mathrm{O}_{5} \\
\quad \mathrm{Do} . \\
\mathrm{V}_{2} \mathrm{O}_{5_{\mathrm{ss}}}{ }^{\mathrm{d}}+\mathrm{V}_{2} \mathrm{O}_{5} \cdot 9 \mathrm{Nb}_{2} \mathrm{O}_{5} \\
\mathrm{~V}_{2} \mathrm{O}_{58 s}{ }^{\mathrm{d}}+\mathrm{V}_{2} \mathrm{O}_{5} \cdot 9 \mathrm{Nb}_{2} \mathrm{O}_{5}+\text { unknown }^{-c} \\
\text { Glass } \\
\text { Do. }\end{array}$ \\
\hline 85 & 15 & $\begin{array}{r}\text { f } 640 \\
\text { f } 652 \\
658 \\
665 \\
\text { f } 666 \\
670 \\
674 \\
688 \\
\\
746 \\
828 \\
902 \\
961 \\
1011 \\
1061 \\
1096\end{array}$ & $\begin{array}{l}.33 \\
.33 \\
21.33 \\
16 \\
.5 \\
.5 \\
.5 \\
1^{.5} \\
{ }^{4} \\
{ }^{1} .5 \\
.5\end{array}$ & $\begin{array}{l}\text { Not melted__. } \\
\text { Partially melted } \\
\text { Partially melted } \\
\text { do do }\end{array}$ & $\begin{array}{l}\mathrm{V}_{2} \mathrm{O}_{5}+\mathrm{V}_{2} \mathrm{O}_{5} \cdot 9 \mathrm{Nb}_{2} \mathrm{O}_{5}+\text { unknown c } \\
\mathrm{V}_{2} \mathrm{O}_{588} \mathrm{~d}+\mathrm{V}_{2} \mathrm{O}_{5} \cdot 9 \mathrm{Nb}_{2} \mathrm{O}_{5}+\text { unknown } \\
\text { Do. } \\
\text { Do. } \\
\mathrm{V}_{2} \mathrm{O}_{5_{88}} \mathrm{~d}+\mathrm{V}_{2} \mathrm{O}_{5} \cdot 9 \mathrm{Nb}_{2} \mathrm{O}_{5} \\
\mathrm{~V}_{2} \mathrm{O}_{5_{88}} \mathrm{~d}+\mathrm{V}_{2} \mathrm{O}_{5} \cdot 9 \mathrm{Nb}_{2} \mathrm{O}_{5} \\
\mathrm{~V}_{2} \mathrm{O}_{5} \mathrm{~d}_{88}+\mathrm{V}_{2} \mathrm{O}_{5} \cdot 9 \mathrm{Nb}_{2} \mathrm{O}_{5} \\
\text { Do. } \\
\text { Do. } \\
\text { Do. } \\
\text { Do. } \\
\text { Glass } \\
\text { Do. }\end{array}$ \\
\hline
\end{tabular}


TABLE 4. Experimental data for compositions in the system vanadium oxide-niobium oxide-Continued

\begin{tabular}{|c|c|c|c|c|c|}
\hline \multicolumn{2}{|c|}{ Composition } & \multicolumn{2}{|c|}{ Heat treatment a } & \multicolumn{2}{|r|}{ Results } \\
\hline $\mathrm{V}_{2} \mathrm{O}_{5}$ & $\mathrm{Nb}_{2} \mathrm{O}_{5}$ & Temp. & Time & Physical observation & $\mathrm{X}$-ray diffraction analysis $b$ \\
\hline $\begin{array}{l}\text { Mole } \% \\
80\end{array}$ & $\begin{array}{c}\text { Mole } \% \\
20\end{array}$ & $\begin{array}{l}{ }^{\circ} \mathrm{C} \\
1061 \\
1119 \\
1140\end{array}$ & $\begin{array}{l}h r \\
1 \\
\quad .75 \\
.5\end{array}$ & $\begin{array}{l}\text { Partially melted } \\
\text { Completely melted. }\end{array}$ & $\begin{array}{l}\mathrm{V}_{2} \mathrm{O}_{5} \mathrm{~d}_{88}+\mathrm{V}_{2} \mathrm{O}_{5} \cdot 9 \mathrm{Nb}_{2} \mathrm{O}_{5} \\
\text { Glass } \\
\text { Do. }\end{array}$ \\
\hline 75 & 25 & $\begin{array}{l}\mathrm{f} 650 \\
\mathrm{f} 690 \\
1061 \\
1130 \\
1154 \\
1180\end{array}$ & $\begin{array}{c}1 \\
16 \\
1 \\
.5 \\
.5 \\
.33\end{array}$ & $\begin{array}{l}\text { Partially melted } \\
\text { Partially melted } \\
\text { Completely melted } \\
\text { do do }\end{array}$ & $\begin{array}{l}\mathrm{V}_{2} \mathrm{O}_{5}^{\mathrm{d}_{88}}+\mathrm{V}_{2} \mathrm{O}_{5} \cdot 9 \mathrm{Nb}_{2} \mathrm{O}_{5} \\
\text { Do. } \\
\text { Do. } \\
\text { Glass+ } \\
\text { Glass }\end{array}$ \\
\hline 70 & 30 & $\begin{array}{r}632 \\
\text { f } 670 \\
1064 \\
1175 \\
1204 \\
1235 \\
1246 \\
1257\end{array}$ & $\begin{array}{l}168 \\
\quad .5 \\
.33 \\
.5 \\
.5 \\
.5 \\
.17 \\
.5 \\
.5\end{array}$ & 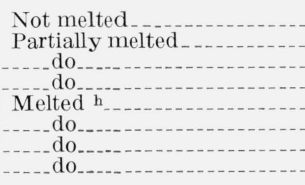 & $\begin{array}{l}\mathrm{V}_{2} \mathrm{O}_{5}+\mathrm{V}_{2} \mathrm{O}_{5} \cdot 9 \mathrm{Nb}_{2} \mathrm{O}_{5}+\text { unknowne, e } \\
\mathrm{V}_{2} \mathrm{O}_{5} \mathrm{~d}_{88}+\mathrm{V}_{2} \mathrm{O}_{5} \cdot 9 \mathrm{Nb}_{2} \mathrm{O}_{5} \\
\mathrm{~V}_{2} \mathrm{O}_{5} \mathrm{~d}_{\mathrm{sg}}+\mathrm{V}_{2} \mathrm{O}_{5} \cdot 9 \mathrm{Nb}_{2} \mathrm{O}_{5}+\text { glass } \\
\mathrm{Glass}^{2}+\text { unknown } \\
\text { Do. } \\
\text { Do. } \\
\text { Do. }\end{array}$ \\
\hline 66.67 & 33.33 & $\begin{array}{l}1045 \\
1064\end{array}$ & ${ }^{3} .66$ & Partially melted & $\begin{array}{l}\mathrm{V}_{2} \mathrm{O}_{5} \mathrm{~d}_{\mathrm{ss}}+\mathrm{V}_{2} \mathrm{O}_{5} \cdot 9 \mathrm{Nb}_{2} \mathrm{O}_{5}+\text { unknown } \\
\mathrm{V}_{2} \mathrm{O}_{5} \mathrm{~d}_{88}+\mathrm{V}_{2} \mathrm{O}_{5} \cdot 9 \mathrm{Nb}_{2} \mathrm{O}_{5}\end{array}$ \\
\hline 65 & 35 & $\begin{array}{l}\text { g } 637 \\
\text { g } 658 \\
1062 \\
1175 \\
1225 \\
1255 \\
1279 \\
1280 \\
\text { f } 654\end{array}$ & $\begin{array}{l}.5 \\
.5 \\
24 \\
.5 \\
.5 \\
.75 \\
.5 \\
.75\end{array}$ & $\begin{array}{l}\text { Not melted } \\
\text { Partially melted } \\
\text { do } \\
\text { Melted } \\
\text { do }{ }^{\mathrm{h}} \\
\text { do } \\
\text { do }\end{array}$ & $\begin{array}{l}\mathrm{V}_{2} \mathrm{O}_{5}+\mathrm{V}_{2} \mathrm{O}_{5} \cdot 9 \mathrm{Nb}_{2} \mathrm{O}_{5} \\
\mathrm{~V}_{2} \mathrm{O}_{5} \mathrm{~d}_{\text {ss }}+\mathrm{V}_{2} \mathrm{O}_{5} \cdot 9 \mathrm{Nb}_{2} \mathrm{O}_{5} \\
\text { Do. } \\
\text { Do. } \\
\text { Do. } \\
\text { Do. } \\
\text { Do. } \\
\text { Do. }\end{array}$ \\
\hline \multirow{5}{*}{60} & & f 684 & 60 & do & $\mathrm{V}_{2} \mathrm{O}_{5} \cdot 9 \mathrm{Nb}_{2} \mathrm{O}_{5}+\mathrm{V}_{2} \mathrm{O}_{0_{\mathrm{es}}}^{\mathrm{d}}$ \\
\hline & & $\begin{array}{l}\text { f } 1014 \\
\text { f } 1062 \\
\text { f } 1100\end{array}$ & $\begin{array}{l}60 \\
24 \\
65\end{array}$ & 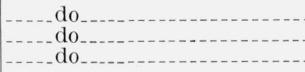 & $\begin{array}{l}\text { Do. } \\
\text { Do. } \\
\text { Do. }\end{array}$ \\
\hline & & ${ }^{\text {f }} 1219$ & .5 & Melted $h_{-} \ldots \ldots . .$. & $\mathrm{V}_{2} \mathrm{O}_{5} \cdot 9 \mathrm{Nb}_{2} \mathrm{O}_{5}+\mathrm{V}_{2} \mathrm{O}_{5 s s}^{\mathrm{d}}$ \\
\hline & & f 1246 & .17 & 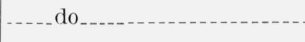 & $\mathrm{V}_{2} \mathrm{O}_{5} \cdot 9 \mathrm{Nb}_{2} \mathrm{O}_{5}+\mathrm{V}_{2} \mathrm{O}_{58 s}^{\mathrm{d}}+$ unknowne \\
\hline & & f 1277 & .08 & do do & $\mathrm{V}_{2} \mathrm{O}_{5} \cdot 9 \mathrm{Nb}_{2} \mathrm{O}_{5}+\mathrm{V}_{2} \mathrm{O}_{88}^{\mathrm{d}}$ \\
\hline 55 & 45 & $\begin{array}{l}1060 \\
1352\end{array}$ & ${ }^{60} .17$ & $\begin{array}{l}\text { Partially melted.-..- } \\
\text { Completely melted..- }\end{array}$ & Do. \\
\hline \multirow[t]{6}{*}{50} & 50 & $\begin{array}{l}600 \\
657\end{array}$ & $\begin{array}{l}6 \\
3\end{array}$ & $\begin{array}{l}\text { Not melted } \\
\text { Partially melted...- }\end{array}$ & $\mathrm{V}_{2} \mathrm{O}_{5} \cdot 9 \mathrm{Nb}_{2} \mathrm{O}_{5}+\mathrm{V}_{2} \mathrm{O}_{5}$ \\
\hline & & f 670 & .5 & - & $\mathrm{V}_{2} \mathrm{O}_{5} \cdot 9 \mathrm{Nb}_{2} \mathrm{O}_{5}+\mathrm{V}_{2} \mathrm{O}_{5_{8 s}}^{\mathrm{d}}$ \\
\hline & & $\begin{array}{r}\mathrm{f} 688 \\
703\end{array}$ & $1^{.5}$ & -_do do & \\
\hline & & 730 & 1 & - $\mathrm{do}_{-}$ & $\mathrm{V}_{2} \mathrm{O}_{5} \cdot 9 \mathrm{Nb}_{2} \mathrm{O}_{5}+\mathrm{V}_{2} \mathrm{O}_{588}^{\mathrm{d}}$ \\
\hline & & \begin{tabular}{r|}
901 \\
999 \\
1049 \\
1092 \\
1300 \\
1358 \\
1382
\end{tabular} & \begin{tabular}{c|}
1 \\
16 \\
22 \\
1.5 \\
.08 \\
.17 \\
.17
\end{tabular} & 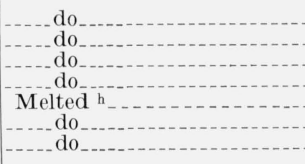 & $\begin{array}{l}\text { Do. } \\
\text { Do. } \\
\text { Do. } \\
\text { Do. } \\
\text { Do. }\end{array}$ \\
\hline & & 1402 & .17 & -.... do & $\mathrm{V}_{2} \mathrm{O}_{5} \cdot 9 \mathrm{Nb}_{2} \mathrm{O}_{5}+\mathrm{V}_{2} \mathrm{O}_{5 s s}^{\mathrm{d}}$ \\
\hline 45 & 55 & $\begin{array}{l}1325 \\
1352\end{array}$ & .1 & - do & \\
\hline 40 & 60 & $\begin{array}{l}1324 \\
1349\end{array}$ & .1 & Melted ${ }^{\mathrm{h}}$ & \\
\hline \multirow[t]{3}{*}{30} & 70 & $\begin{array}{r}963 \\
1119\end{array}$ & $\begin{array}{l}.08 \\
.08\end{array}$ & Partially melted. & $\begin{array}{l}\mathrm{V}_{2} \mathrm{O}_{5} \cdot 9 \mathrm{Nb}_{2} \mathrm{O}_{5}+\mathrm{V}_{2} \mathrm{O}_{5^{85}}^{\mathrm{d}} \\
\text { Do. }\end{array}$ \\
\hline & & 1350 & .08 & Melted $\mathrm{h}_{\ldots}$ & $\mathrm{M}-\mathrm{Nb}_{2} \mathrm{O}_{5}^{\mathrm{i}, \mathrm{e}}$ \\
\hline & & 1375 & .08 & do $h_{\ldots}$ & Do. \\
\hline \multirow[t]{5}{*}{25} & 75 & $\begin{array}{l}629 \\
642\end{array}$ & $\begin{array}{l}.25 \\
.33\end{array}$ & Not melted & $\mathrm{M}-\mathrm{Nb}_{2} \mathrm{O}_{5}{ }^{\mathrm{i}}+\mathrm{L} \mathrm{Nb_{2 } \mathrm { O } _ { 5 }}$ \\
\hline & & 649 & .5 & Partially melted......... & $\mathrm{V}_{2} \mathrm{O}_{5} \cdot 9 \mathrm{Nb}_{2} \mathrm{O}_{5}+\mathrm{V}_{2} \mathrm{O}_{\text {ṡss }}^{\mathrm{d}}+$ unknown。 \\
\hline & & f 767 & .3 & -_-_do & $\mathrm{V}_{2} \mathrm{O}_{5} \cdot 9 \mathrm{Nb}_{2} \mathrm{O}_{5}+\mathrm{V}_{2} \mathrm{O}_{58 \mathrm{sB}}^{\mathrm{d}}$ \\
\hline & & $\begin{array}{r}880 \\
970 \\
\text { g } 1375 \\
\text { g } 1382 \\
\text { g } 1395\end{array}$ & $\begin{array}{r}16 \\
2 \\
.08 \\
.08 \\
.08\end{array}$ & \begin{tabular}{|c|} 
do \\
do \\
do
\end{tabular} & $\begin{array}{l}\text { Do. } \\
\text { Do. }\end{array}$ \\
\hline & & g 1410 & .25 & |____-_do & $\mathrm{L}-\mathrm{Nb}_{2} \mathrm{O}_{5}^{\mathrm{j}}$ \\
\hline
\end{tabular}

See footnotes at end of table. 
TABLE 4. Experimental data for compositions in the system vanadium oxide-niobium oxide-Continued

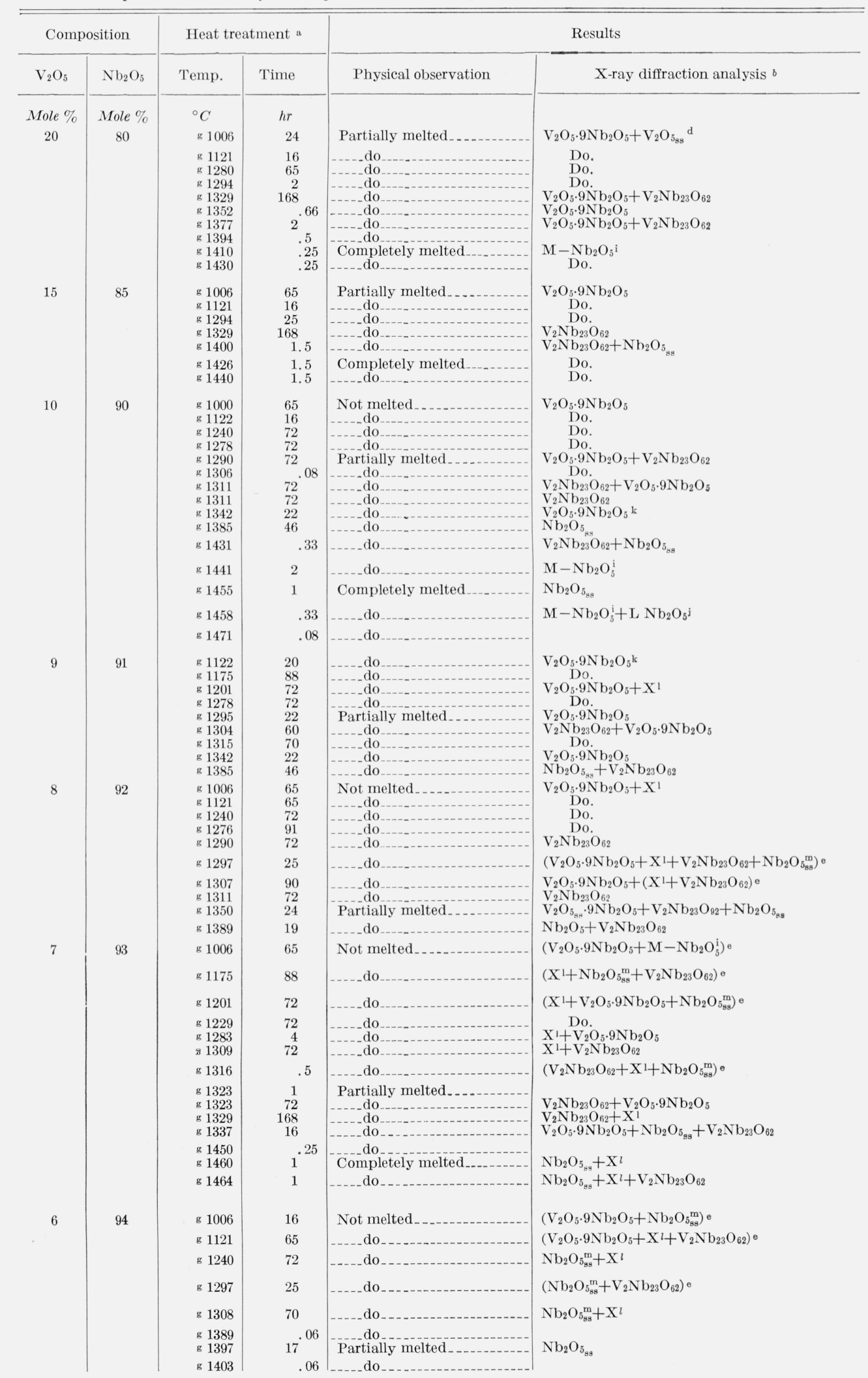

See footnotes at end of table. 
TABLE 4. Experimental data for compositions in the system vanadium oxide-niobum oxide-Continued

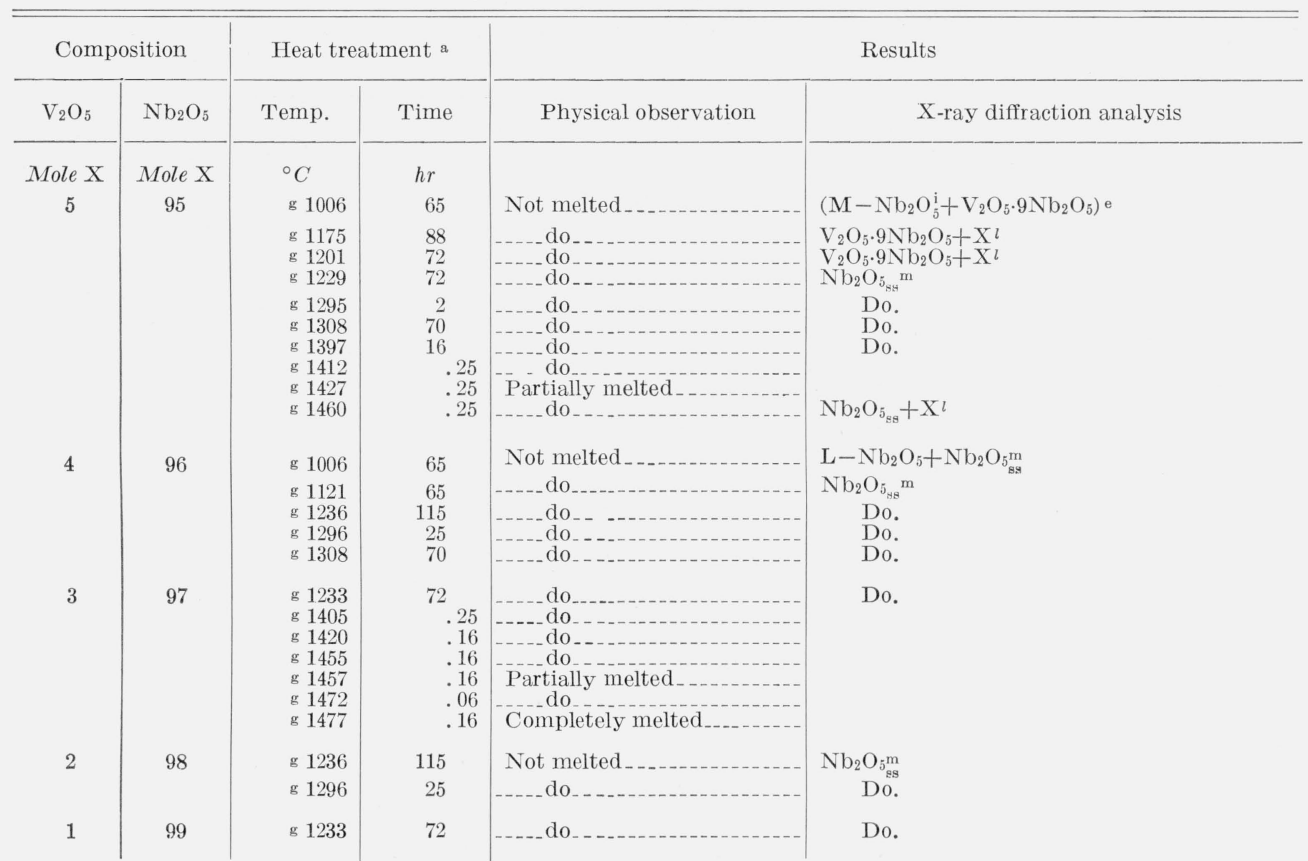

a All specimens were preheated to $500{ }^{\circ} \mathrm{C}$ for $10 \mathrm{hr}$. Rate of heating and cooling was approximately $3 \% \mathrm{~min}$. Specimens were heated in sealed Pt tubes and quenched from temperature indicated.

$\mathrm{b}$ The phases identified are given in the order of the amount present (greatest amount first) at room temperature. These phases are not necessarily those present at the temperature to which the specimen was heated.

present at the temperature to which the specimen was heated.
c Unknown - a small amount of an unknown metastable phase with $\mathrm{x}$-ray c Unknown- a small amount of an unknown metastable phase with X-ray
diffraction lines occurring at the following $2 \theta$ values: $22.22^{\circ}, 27.27^{\circ}, 30.28^{\circ}, 37.84^{\circ}$, $44.78^{\circ}, 45.22^{\circ}, 47.34^{\circ}$ and $49.70^{\circ}$

d Specimen contained metastable " $\mathrm{V}_{2} \mathrm{O}_{5}$ type solid solution" when quenched from above the solidus and examined at room temperature.

e The interpretation of the x-ray diffraction pattern is inconsistent with the phase diagram and is considered to be non-equilibrium due to differences in time and/or temperature of heat treatment. f Second heat treatment was $600{ }^{\circ} \mathrm{C}$ for $168 \mathrm{hr}$ in sealed $\mathrm{Pt}$ tubes. Rate of heating and cooling was approximately $3 \% \mathrm{~min}$.

$\mathrm{g}$ Second heat treatment was $1000^{\circ} \mathrm{C}$ for $5 \mathrm{hr}$. Rate of heating and cooling was approximately $3^{\circ} \mathrm{min}$

h Criteria could not be established to determine complete melting (see sec. 2).

i Specimen contained (nonequilibrium) M-type $\mathrm{Nb}_{2} \mathrm{O}_{5}[29]$.

ed (nonequilibrium) M-type $\mathrm{Nb}_{2} \mathrm{O}_{5}[29]$. Specimen contained (nonequilibrium) low temperature form of $\mathrm{N}$

uenched from above the liquidus and examined at room ten perature.

$\mathrm{k}$ The presence of a second phase was detected with a petrographic

${ }^{l}$ Unknown phase occurring at about 93 to 94 mole percent $\mathrm{Nb}_{2} \mathrm{O}_{5}$.

$\mathrm{m}$ Specimen contained a phase interpreted as $\mathrm{Nb}_{2} \mathrm{O}_{5}$ solid solution, however other discrete phases may be present which are not detected by the present ex. perimental methods.

\subsection{Description of Phase Diagram}

The liquidus falls sharply from the melting point of $\mathrm{V}_{2} \mathrm{O}_{5}\left(675^{\circ} \mathrm{C}\right)$ to a eutectic at about 1 mole percent $\mathrm{Nb}_{2} \mathrm{O}_{5}$ and $648^{\circ} \mathrm{C}$. The exact eutectic composition was not determined because of the relatively small amount of $\mathrm{Nb}_{2} \mathrm{O}_{5}$ involved and the liquidus lines defining the eutectic composition are dashed. The liquidus is also dashed from 30 to 70 mole percent $\mathrm{Nb}_{2} \mathrm{O}_{5}$ (see sec. 2) and is indicated as rising smoothly from the eutectic composition to a peritectic at about 48 mole percent $\mathrm{Nb}_{2} \mathrm{O}_{5}$ and $1290{ }^{\circ} \mathrm{C}$ which corresponds to the incongruent melting temperature of the compound $\mathrm{V}_{2} \mathrm{O}_{5} \cdot 9 \mathrm{Nb}_{2} \mathrm{O}_{5}$. There is no apparent solid solution on either side of this compound, as indicated by the similarity of unit cell dimensions in the two phase regions adjacent to the $1: 9$ composition. The liquidus again rises smoothly to another peritectic at about 57 mole percent $\mathrm{Nb}_{2} \mathrm{O}_{5}$ and $1322{ }^{\circ} \mathrm{C}$ which represents the incongruent melting temperature of the compound $\mathrm{V}_{2} \mathrm{Nb}_{23} \mathrm{O}_{62}$.
A two phase area should separate the compounds $\mathrm{V}_{2} \mathrm{Nb}_{23} \mathrm{O}_{62}$ and $\mathrm{V}_{2} \mathrm{O}_{5} \cdot 9 \mathrm{Nb}_{2} \mathrm{O}_{5}$. This small area, represented in figures 1 and 2 as extending in width from 90 to 92 mole percent $\mathrm{Nb}_{2} \mathrm{O}_{5}$, was not found experimentally and is dashed. The liquidus rises again from approximately 60 mole $\% \mathrm{Nb}_{2} \mathrm{O}_{5}$ and $1332{ }^{\circ} \mathrm{C}$, the incongruent melting point of the phase occurring at some composition apparently between 93 and 94 mole percent $\mathrm{Nb}_{2} \mathrm{O}_{5}$ to the melting point of $\mathrm{Nb}_{2} \mathrm{O}_{5}$.

A narrow two phase area was found to occur from about 92 to 94 mole percent $\mathrm{Nb}_{2} \mathrm{O}_{5}$ separating the $\mathrm{X}$ compound and the $2 \mathrm{~V}_{2} \mathrm{O}_{5} \cdot 23 \mathrm{Nb}_{2} \mathrm{O}_{5}$ compound. Another two phase area, of less than 1 mole percent width just below the solidus, separates the $\mathrm{X}$ compound and $\mathrm{Nb}_{2} \mathrm{O}_{5}$ ss field. About 5 mole percent $\mathrm{V}_{2} \mathrm{O}_{5}$ is apparently accepted by $\mathrm{Nb}_{2} \mathrm{O}_{5}$ in solid solution. Since the unit cell dimensions of the $\mathrm{Nb}_{2} \mathrm{O}_{5}$ solid solution did not change significantly with composition, the solid solution area was delineated by the disappearing phase method. In addition, it should again be emphasized at this 
point, that the $\mathrm{x}$-ray diffraction patterns for $2 \mathrm{~V}_{2} \mathrm{O}_{5}: 23 \mathrm{Nb}_{2} \mathrm{O}_{5}$ and the $\mathrm{X}$ compound bear a marked similarity to one another and to the high temperature form of $\mathrm{Nb}_{2} \mathrm{O}_{5}$. Furthermore, it is not impossible that other discrete phases may exist which are below the limit of detection of the experimental conditions employed.

Since $\mathrm{V}_{2} \mathrm{O}_{5}$ did not stabilize the low temperature form of $\mathrm{Nb}_{2} \mathrm{O}_{5}$ and since temperatures below $1200{ }^{\circ} \mathrm{C}$ were not sufficient to attain equilibrium, the lines delineating the areas represented on figure 1 from 90 to 99 mole percent $\mathrm{Nb}_{2} \mathrm{O}_{5}$ (below $1200{ }^{\circ} \mathrm{C}$ ) were dashed.

The liquidus rises again from $1332{ }^{\circ} \mathrm{C}$ and 60 mole percent $\mathrm{Nb}_{2} \mathrm{O}_{5}$, the peritectic for the $\mathrm{X}$ compound to the melting point of $\mathrm{Nb}_{2} \mathrm{O}_{5}$. This $1485{ }^{\circ} \mathrm{C}$ melting point was determined previously by the authors [22] and was not redetermined for this study.

\subsection{Reversibility, Oxidation, and Reduction}

Relatively long periods of time at temperatures above the apparent minimum decomposition temperature of $1288{ }^{\circ} \mathrm{C}$ were required in order to form the $2 \mathrm{~V}_{2} \mathrm{O}_{5}: 23 \mathrm{Nb}_{2} \mathrm{O}_{5}$ compound. This apparent minimum decomposition was delineated from data obtained by heating the compound at successively higher temperatures.

To establish reversibility, it is necessary to decompose the compound into the same two phases previously seen below the apparent minimum decomposition temperature. Both the 92 and 93 mole percent $\mathrm{Nb}_{2} \mathrm{O}_{5}$ compositions were reheated at lower temperatures in sealed and unsealed Pt tubes and these results are given in table 5 . The composition 8 mole percent $\mathrm{V}_{2} \mathrm{O}_{5}: 92$ mole percent $\mathrm{Nb}_{2} \mathrm{O}_{5}$ when heated to $1307{ }^{\circ} \mathrm{C}$ for $90 \mathrm{hr}$ in a sealed $\mathrm{Pt}$ tube formed the compound $\mathrm{V}_{2} \mathrm{Nb}_{23} \mathrm{O}_{62}$. This compound when reheated in a closed $\mathrm{Pt}$ tube at $1222{ }^{\circ} \mathrm{C}$ for 70 hr as outlined in table 5 was not reversed to $\mathrm{V}_{2} \mathrm{O}_{5} \cdot 9 \mathrm{Nb}_{2} \mathrm{O}_{5}+\mathrm{X}$ phase, but formed only the $\mathrm{X}$ phase at the expense of some $\mathrm{V}_{2} \mathrm{Nb}_{23} \mathrm{O}_{62}$. This $\mathrm{X}$ phase apparently exists at some composition between 93 and 94 mole percent $\mathrm{Nb}_{2} \mathrm{O}_{5}$. A portion of the same specimen previously heated $1222{ }^{\circ} \mathrm{C}$ for $70 \mathrm{hr}$ was again reheated to $1298{ }^{\circ} \mathrm{C}$ for $68 \mathrm{hr}$ in a sealed $\mathrm{Pt}$ tube and further reduction apparently took place. Some of the $\mathrm{V}_{2} \mathrm{Nb}_{23} \mathrm{O}_{62}$ was still present but the $\mathrm{X}$ phase could no longer be detected. Another more reduced phase occurred which was found from $\mathrm{x}$-ray diffraction data to be apparently isostructural with the compound $\mathrm{Ti}_{2} \mathrm{Nb}_{10} \mathrm{O}_{29}$ reported by Wadsley [23].

The situation, however, is quite different when the same composition received a similar heat treatment in unsealed $\mathrm{Pt}$ tubes. When the compound $\mathrm{V}_{2} \mathrm{Nb}_{23} \mathrm{O}_{62}$, previously formed by heating to $1307{ }^{\circ} \mathrm{C}$ for $90 \mathrm{hr}$ was reheated at $1222{ }^{\circ} \mathrm{C}$ for $70 \mathrm{hr}$ in an unsealed tube, the x-ray diffraction powder pattern data indicated that the specimen apparently contained a non-equilibrium mixture of three phases, the $\mathrm{X}$ phase, $\mathrm{V}_{2} \mathrm{O}_{5} \cdot 9 \mathrm{Nb}_{2} \mathrm{O}_{5}$, and $\mathrm{V}_{2} \mathrm{Nb}_{23} \mathrm{O}_{62}$. Apparently it was approaching binary equilibrium and tending to reverse into $\mathrm{V}_{2} \mathrm{O}_{5} \cdot 9 \mathrm{Nb}_{2} \mathrm{O}_{5}+\mathrm{X}$ phase. A portion of this material previously heated at $1222{ }^{\circ} \mathrm{C}$ for $70 \mathrm{hr}$ unsealed was again reheated to $1298{ }^{\circ} \mathrm{C}$ for $68 \mathrm{hr}$ in an open tube. The $\mathrm{x}$-ray diffraction pattern of this material showed the presence of 2 phases $2 \mathrm{~V}_{2} \mathrm{O}_{5} \cdot 23 \mathrm{Nb}_{2} \mathrm{O}_{5}$ and $\mathrm{X}$ phase. It has apparently changed composition slightly due to volatilization.

In view of the foregoing discussion of an open versus closed system, it can probably be concluded that in the $\mathrm{V}_{2} \mathrm{O}_{5}-\mathrm{Nb}_{2} \mathrm{O}_{5}$ system, specimens when

TABLE 5. Experimental data for reversibility of phases in the system vanadium oxide-niobium oxide

\begin{tabular}{|c|c|c|c|c|c|c|c|}
\hline \multicolumn{2}{|c|}{ Composition Mole \% } & \multicolumn{2}{|c|}{ Initial heat treatment } & \multirow{2}{*}{$\begin{array}{c}\text { X-ray a } \\
\text { Identification }\end{array}$} & \multicolumn{2}{|c|}{ Final heat treatment } & \multirow{2}{*}{$\frac{\text { X-ray a }}{\text { Identification }}$} \\
\hline $\mathrm{V}_{2} \mathrm{O}_{5}$ & $\mathrm{Nb}_{2} \mathrm{O}_{5}$ & Temp. & Time & & Temp. & Time & \\
\hline \multirow{3}{*}{8} & 93 & $\begin{array}{r}{ }^{\circ} C \\
500 \\
1000 \\
\text { b } 1323 \\
500 \\
1000 \\
\text { b } 1323 \\
\end{array}$ & $\begin{array}{rr} \\
h r \\
& 10 \\
& 5 \\
& 72 \\
& 10 \\
& 5 \\
& 52 \\
& \end{array}$ & $\begin{array}{l}\mathrm{X}^{\mathrm{c}}+\mathrm{V}_{2} \mathrm{O}_{5} \cdot 9 \mathrm{Nb}_{2} \mathrm{O}_{5} \\
\mathrm{X}^{\mathrm{c}}+\mathrm{V}_{2} \mathrm{O}_{5} \cdot 9 \mathrm{Nb}_{2} \mathrm{O}_{5}\end{array}$ & $\begin{array}{l}{ }^{\circ} \mathrm{C} \\
\text { b } 1235 \\
{ }^{\text {b } 1219}\end{array}$ & 72 & $\begin{array}{l}\mathrm{X} c \\
\mathrm{X}^{\mathrm{c}}+\mathrm{V}_{2} \mathrm{O}_{5} \cdot 9 \mathrm{Nb}_{2} \mathrm{O}_{5}\end{array}$ \\
\hline & 92 & $\begin{array}{r}500 \\
\text { b } 1307 \\
500 \\
\text { b } 1307 \\
500 \\
\text { b } 1307 \\
\text { b } 1222\end{array}$ & $\begin{array}{l}10 \\
90 \\
10 \\
90 \\
10 \\
90 \\
70 \\
\end{array}$ & $\begin{array}{l}2 \mathrm{~V}_{2} \mathrm{O}_{5} \cdot 23 \mathrm{Nb}_{2} \mathrm{O}_{5} \ldots \ldots \\
2 \mathrm{~V}_{2} \mathrm{O}_{5} \cdot 23 \mathrm{Nb}_{2} \mathrm{O}_{5} \\
2 \mathrm{~V}_{2} \mathrm{O}_{5} \cdot 23 \mathrm{Nb}_{2} \mathrm{O}_{5}+\mathrm{Xc}^{\mathrm{c}}\end{array}$ & $\begin{array}{l}\text { b } 1298 \\
\text { b } 1222 \\
\text { b } 1298\end{array}$ & $\begin{array}{r}168 \\
70 \\
68\end{array}$ & $\begin{array}{l}2 \mathrm{~V}_{2} \mathrm{O}_{5} \cdot 23 \mathrm{Nb}_{2} \mathrm{O}_{5} \\
2 \mathrm{~V}_{2} \mathrm{O}_{5} \cdot 23 \mathrm{Nb}_{2} \mathrm{O}_{5}+\mathrm{X}^{\mathrm{e}} \\
2 \mathrm{~V}_{2} \mathrm{O}_{5} \cdot 23 \mathrm{Nb}_{2} \mathrm{O}_{5}+(\mathrm{V}, \mathrm{Nb})_{12} \mathrm{O}_{29} \mathrm{~d}\end{array}$ \\
\hline & & $\begin{array}{r}500 \\
\text { b } 1307 \\
500 \\
\text { e } 1307 \\
\text { e } 1222\end{array}$ & $\begin{array}{l}10 \\
90 \\
10 \\
90 \\
70\end{array}$ & $\begin{array}{l}2 \mathrm{~V}_{2} \mathrm{O}_{5} \cdot 23 \mathrm{Nb}_{2} \mathrm{O}_{5} \\
\mathrm{X}^{\mathrm{c}}+\mathrm{V}_{2} \mathrm{O}_{5} \cdot 9 \mathrm{Nb}_{2} \mathrm{O}_{5}+2 \mathrm{~V}_{2} \mathrm{O}_{5} \cdot 23 \mathrm{Nb}_{2} \mathrm{O}_{5}\end{array}$ & e 1222 & 70 & $\begin{array}{l}\mathrm{X}^{\mathrm{c}}+\mathrm{V}_{2} \mathrm{O}_{5} \cdot 9 \mathrm{Nb}_{2} \mathrm{O}_{5}+2 \mathrm{~V}_{2} \mathrm{O}_{5} \cdot 23 \mathrm{Nb}_{2} \mathrm{O}_{5} \\
2 \mathrm{~V}_{2} \mathrm{O}_{5} \cdot 23 \mathrm{Nb}_{2} \mathrm{O}_{5}\end{array}$ \\
\hline
\end{tabular}

a The phases identified are given in the order of amount present (greatest amount first) at room temperature. The phases are not necessarily those present at the temperature to which the specimens were heated.

$\mathrm{b}$ Specimen heated in sealed Pt tube and quenched.

c Unknown phase occurring in the system at approximately 93 to 94 mole percent $\mathrm{Nb}_{2} \mathrm{O}_{5}$.
dA reduced phase apparently isostructural with the monoclinic form of $2 \mathrm{TiO}_{2} \cdot 5 \mathrm{Nb}_{2} \mathrm{O}_{5}[23]$.

e Specimen heated in open Pt tube and quenched. 
heated in a closed or oxygen deficient environment tended to reduce with time and those heated in an open or oxygen rich environment tended to reverse and approach binary equilibrium. Since the experimental evidence apparently did not validate reversibility in the closed or restricted oxygen situation, the line indicating a minimum decomposition for the compound $\mathrm{V}_{2} \mathrm{Nb}_{23} \mathrm{O}_{62}$ was dashed.

\section{5. $\mathrm{Nb}_{2} \mathrm{O}_{5}$-Type "Solid Solution"}

A re-examination of selected compositions in the systems $\quad \mathrm{ZnO}-\mathrm{Nb}_{2} \mathrm{O}_{5} ; \quad \mathrm{NiO}-\mathrm{Nb}_{2} \mathrm{O}_{5} ; \quad \mathrm{Al}_{2} \mathrm{O}_{3}-\mathrm{Nb}_{2} \mathrm{O}_{5}$; $\mathrm{TiO}_{2}-\mathrm{Nb}_{2} \mathrm{O}_{5}$; and $\mathrm{ZrO}_{2}-\mathrm{Nb}_{2} \mathrm{O}_{5}$, has shown that phases reported previously as " $\mathrm{Nb}_{2} \mathrm{O}_{5}$ solid solution" are, in fact, discrete compounds. Several of the compounds in the $\mathrm{V}_{2} \mathrm{O}_{5}-\mathrm{Nb}_{2} \mathrm{O}_{5}$ pseudo-binary system ( $\mathrm{VNb}_{9} \mathrm{O}_{25}, \mathrm{~V}_{2} \mathrm{Nb}_{23} \mathrm{O}_{62}$ and $\left.(\mathrm{V}, \mathrm{Nb}){ }_{12} \mathrm{O}_{29}\right)$ have apparently analogous structures in other mixed oxide systems.

All of these phases are grouped in table 6 into five general formula types according to the cation to anion ratio.

It is worth noting that in this investigation no phase was detected in the $\mathrm{V}_{2} \mathrm{O}_{5}-\mathrm{Nb}_{2} \mathrm{O}_{5}$ pseudobinary system with either of the cation to anion ratios $11: 27$ or $3: 7$.

Goldschmidt [3] reported the existence of $\mathrm{Nb}_{2} \mathrm{O}_{5 \mathrm{~s}}$ in the $\mathrm{Al}_{2} \mathrm{O}_{3}-\mathrm{Nb}_{2} \mathrm{O}_{5}$ system. Thereafter, Layden [24] postulated from the Norin and Magneli [25] formulae that compounds should occur at the compositions $\mathrm{Al}_{2} \mathrm{O}_{3} \cdot 9 \mathrm{Nb}_{2} \mathrm{O}_{5}, \mathrm{Al}_{2} \mathrm{O}_{3} \cdot 25 \mathrm{Nb}_{2} \mathrm{O}_{5}$ and $\mathrm{Al}_{2} \mathrm{O}_{3}$. $49 \mathrm{Nb}_{2} \mathrm{O}_{5}$ but found only the first two. Roth, Wadsley \& Gatehouse [26] reported the compound $\mathrm{Al}_{\frac{1}{2}} \mathrm{Nb}_{24 \frac{1}{2}} \mathrm{O}_{62} \quad(1: 49)$ and indicated, from structural considerations, that the 1:25 compound should have the composition $\mathrm{AlNb}_{21} \mathrm{O}_{54}$. Andersson and Roth [27] from single crystal data found the compound occurring around the 1:9 composition to have the actual formula $\mathrm{Al}_{2} \mathrm{O}_{3} \cdot 11 \mathrm{Nb}_{2} \mathrm{O}_{5}$.

\section{Summary}

The system $\mathrm{V}_{2} \mathrm{O}_{5}-\mathrm{Nb}_{2} \mathrm{O}_{5}$ was studied and the phase diagram was constructed from fusion characteristics, and x-ray diffraction data.

From structural considerations and from experimental evidence this system does not appear to be binary.

There seemed to be no evidence for equilibrium solid solution of $\mathrm{Nb}_{2} \mathrm{O}_{5}$ in $\mathrm{V}_{2} \mathrm{O}_{5}$ and a eutectic occurred at approximately 1 mole percent $\mathrm{Nb}_{2} \mathrm{O}_{5}$ and $648{ }^{\circ} \mathrm{C}$.

The existence of three intermediate compounds was postulated. The first, $\mathrm{V}_{2} \mathrm{O}_{5} \cdot 9 \mathrm{Nb}_{2} \mathrm{O}_{5}$ melted incongruently at about $1290{ }^{\circ} \mathrm{C}$ and the second $\mathrm{V}_{2} \mathrm{Nb}_{23} \mathrm{O}_{62}$ melted incongruently at about $1322{ }^{\circ} \mathrm{C}$. The third compound which melted incongruently at about $1332{ }^{\circ} \mathrm{C}$ was found to exist at some composition between about 93 and 94 mole percent $\mathrm{Nb}_{2} \mathrm{O}_{5}$. Up to about 5 mole percent $\mathrm{V}_{2} \mathrm{O}_{5}$ was apparently accepted by $\mathrm{Nb}_{2} \mathrm{O}_{5}$ in solid solution lowering the melting point of $\mathrm{Nb}_{2} \mathrm{O}_{5}$ from 1485 to $1332{ }^{\circ} \mathrm{C}$.

The possibility of other discrete phases which cannot be identified by the experimental techniques employed in the study must not be excluded in the region from 90 to 99 mole percent $\mathrm{Nb}_{2} \mathrm{O}_{5}$. In addition the $\mathrm{Nb}_{2} \mathrm{O}_{5}$ rich portions of the following binary systems were reinvestigated: $\mathrm{ZnO}-\mathrm{Nb}_{2} \mathrm{O}_{5}$, $\mathrm{NiO}-\mathrm{Nb}_{2} \mathrm{O}_{5}, \quad \mathrm{Al}_{2} \mathrm{O}_{3}-\mathrm{Nb}_{2} \mathrm{O}_{5}, \quad \mathrm{TiO}_{2}-\mathrm{Nb}_{2} \mathrm{O}_{5}$, and $\mathrm{ZrO}_{2}-$ $\mathrm{Nb}_{2} \mathrm{O}_{5}$. Solid solution of $\mathrm{M}_{\mathrm{x}} \mathrm{O}_{\mathrm{y}}$ with $\mathrm{Nb}_{2} \mathrm{O}_{5}$ was previously reported in all cases in excess of 90 mole percent $\mathrm{Nb}_{2} \mathrm{O}_{5}$. Subsequent data (table 6) has shown that in each of these systems discrete compounds could be identified in compositions previously reported as being $\mathrm{Nb}_{2} \mathrm{O}_{5}$ solid solution. 'The compound $\mathrm{V}_{2} \mathrm{Nb}_{23} \mathrm{O}_{62}$ is apparently isostructural with $\mathrm{TiNb}_{24} \mathrm{O}_{62}$ and $\mathrm{Al}_{\frac{1}{2}} \mathrm{Nb}_{24 \frac{1}{2}} \mathrm{O}_{62}$. The highly reduced compound $(\mathrm{V}, \mathrm{Nb})_{12} \mathrm{O}_{29}$ is apparently isostructural with $\mathrm{Ti}_{2} \mathrm{Nb}_{10} \mathrm{O}_{29}, \quad \mathrm{Nb}_{12} \mathrm{O}_{29}, \quad \mathrm{AlNb}_{11} \mathrm{O}_{29}$ $\mathrm{NiNb}_{11} \mathrm{O}_{29}$, and $\mathrm{ZnNb}_{11} \mathrm{O}_{29}$.

TABLE 6. Apparently isostructural phases in mixed oxide systems of $\mathrm{Nb}_{2} \mathrm{O}_{5}$ or $\mathrm{Ta}_{2} \mathrm{O}_{5}$

\begin{tabular}{|c|c|c|c|c|}
\hline \multirow{2}{*}{$\begin{array}{l}\text { Composition } \\
\text { Cation: Anion }\end{array}$} & \multicolumn{4}{|c|}{ Valence of cation additions to $\mathrm{Nb}_{2} \mathrm{O}_{5}$ and/or $\mathrm{Ta}_{2} \mathrm{O}_{5}$} \\
\hline & $\mathrm{M}_{2} \mathrm{O}_{5}-\mathrm{M}_{2}^{\prime} \mathrm{O}_{5}$ & $\mathrm{M} \mathrm{O}_{2}-\mathrm{M}_{2}^{\prime} \mathrm{O}_{5}$ & $\mathrm{M}_{2} \mathrm{O}_{3}-\mathrm{M}_{2}^{\prime} \mathrm{O}_{5}$ & $\mathrm{M} \mathrm{O}-\mathrm{M}_{2}^{\prime} \mathrm{O}_{5}$ \\
\hline 2:5 & $\begin{array}{l}\mathrm{Nb}_{2} \mathrm{O}_{5} \\
\mathrm{~V} \mathrm{Nb} \mathrm{O}_{25} \text { a }[13] \\
\mathrm{P} \mathrm{Nb}_{9} \mathrm{O}_{25}[13] \\
\mathrm{As} \mathrm{N}_{9} \mathrm{O}_{25}[13] \\
\mathrm{Ta}_{3} 1 / 3 \mathrm{Nb}_{6} 2 / 3 \mathrm{O}_{25}[13] \\
\mathrm{P} \mathrm{Ta}_{9} \mathrm{O}_{25}[13] \\
\mathrm{As} \mathrm{Ta9}_{25}[13] \\
\mathrm{V} \mathrm{T} \mathrm{Ta}_{25} \mathrm{O}_{25}[13] \\
\end{array}$ & $\mathrm{GeO}_{2} \cdot 9 \mathrm{Nb}_{2} \mathrm{O}_{5}$ b [13] & & \\
\hline 25:62 & $\mathrm{V}_{22} \mathrm{Nb}_{23} \mathrm{O}_{62} \mathrm{c}+\frac{1}{2} \mathrm{O}$ & $\begin{array}{l}\mathrm{TiNb}_{24} \mathrm{O}_{62} \text { d }[1,16] \\
\mathrm{ZrNb}_{24} \mathrm{O}_{62} \mathrm{~d}[1]\end{array}$ & $\mathrm{Al}_{1,2} \mathrm{Nb}_{24}{ }_{1 / 2} \mathrm{O}_{62}[26]$ & \\
\hline $11: 27$ & $\begin{array}{l}{\left[\mathrm{NbO}_{2 \cdot 46}\right][25]} \\
{\left[\mathrm{NbO}_{2 \cdot 467}\right][28]} \\
{\left[\mathrm{NbO}_{2 \cdot 454}\right][26]}\end{array}$ & & $\begin{array}{l}\mathrm{Al}_{2} \mathrm{O}_{3} \cdot 25 \mathrm{Nb}_{2} \mathrm{O}_{5}[24] \\
\mathrm{Al}_{1 / 2} \mathrm{Nb}_{10}{ }_{1 / 2} \mathrm{O}_{27}\end{array}$ & \\
\hline 12:29 & $(\mathrm{V}, \mathrm{Nb})_{12} \mathrm{O}_{29}+\mathrm{O}$ e & $\mathrm{Ti}_{2} \mathrm{Nb}_{10} \mathrm{O}_{29}[23]$ & $\mathrm{AlNb}_{11} \mathrm{O}_{29}[26][27]$ & $\begin{array}{l}\mathrm{Ni}_{2 / 3} \mathrm{Nb}_{11} 1 / 3 \mathrm{O}_{29} \\
\mathrm{Zn}_{2 / 3} \mathrm{Nb}_{11}{ }_{1 / 3} \mathrm{O}_{29} \mathrm{e}\end{array}$ \\
\hline $3: 7$ & $\mathrm{TiNb}_{2} \mathrm{O}_{7}[23]$ & & & \\
\hline
\end{tabular}

a This phase was previously reported as solid solution [3] (see sect. 3). b $\mathrm{Ge}^{+4}$ is accepted into the structure and would seem to indicate that either the cation/anion ratio is varying or that minor differences exist in the packing of cation coordination polyhedra.

c This phase has not been previously reported in the binary system. d This phase was originally reported as solid solution [1] but later described as a compound by one of the authors [16]

e This phase previously unreported is apparently isostructural with the high temperature polymorph of $\mathrm{Ti}_{2} \mathrm{Nb}_{10} \mathrm{O}_{29}$ and has the following unit cell dimensions: $a=15.57 \AA, b=3.826 \AA$, $c=20.5 \AA$ and $\beta=113^{\circ} 41^{\prime}$. 


\section{References}

[1] R. S. Roth and L. W. Coughanour, J. Res. NBS 55 [4] 209-213 (1955)

[2] A. J. Pollard, J. Am. Ceram. Soc. 44 [12] 630 (1961).

[3] H. J. Goldschmidt (1960), Metallurgia 62, 241.

[4] A. Burdese, M. Borlera, Ric. Sci. Rend. Sez. A3 [7] 1025 (1963).

[5] H. F. Stimson, J. Res. NBS 65A, 139-145 (1960).

[6] H. S. Roberts, Phys. Rev. 23, 386-95 (1924).

[7] R. S. Roth and E. M. Levin; J. Research NBS 62 [5] $193-200(1959)$

[8] F. Holtzberg, A. Reisman, M. Berry, and M. Berkenblit; J. Amer. Chem. Soc. 78 [7] 1536-1540 (1956).

[9] E. M. Levin and C. L. McDaniel; NBS personal communication

[10] A. Bystrom, K. A. Wilhelmi, and O. Brotzen, Act. Chem. Sci. 1119-1130 (1950).

[11] H. E. Swanson, N. T. Gilfrich, M. I. Cook, R. S. Stinchfield, and P. C. Parks, NBS Cir. 539 [8] 66-67 (1959).

[12] C. L. MeDaniel, personal communication.

[13] J. L. Waring and R. S. Roth, Acta Cryst. 17 [4] 455-456 (1964).

[14] F. Holtzberg and A. Reisman, J. Phys. Chem. 65 1193 (1961).
[15] G. P. Mohanty, L. J. Fiegel and J. H. Healy, Acta Cryst. 151190 (1962).

[16] R. S. Roth and A. D. Wadsley, Acta Cryst. (accepted for publication).

[17] F. Holtzberg, A. Reisman, and M. Berry, J. Am. Chem. Soc. 79 2039-2043 (1957).

[18] M. W. Shafer and R. Roy, Z. Krist. 110 [3] 241-244 (1958).

[19] H. J. Goldschmidt, J. Inst. Metals 87 235-239 (1958-59)

[20] A. Reisman and F. Holtzberg, J. Am. Chem. Soc. 81 3182-3184 (1958)

[21] R. S. Roth, J. Research NBS 62, 27 (1959).

[22] R. S. Roth and J. L. Waring, J. Research NBS 66A [6] 451-463 (1962)

[23] A. D. Wadsley, Acta Cryst. 14, 660-670 (1961).

[24] G. K. Layden, J. Amer. Cer. Soc. 46 [10] 506 (1963)

[25] R. Norin and A. Magneli, Naturwiss. 47 [15] 354-55 $(1960)$.

[26] R. S. Roth, A. D. Wadsley, and B. M. Gatehouse, Naturwiss. 51 [11] 262-263 (1964)

[27] S. Andersson and R. S. Roth, personal communication.

[28] R. Gruehn and H. Schäfer, Naturwiss. 50 642 (1963).

[29] G. Brauer, Z. Anorg. u. allgem. Chem. 248, 1 (1941).

(Paper 69A2-333) 